\title{
Rutin induces apoptosis via P53 up-regulation in human glioma CHME cells
}

\author{
Xiaopeng Yan, Yali Hao, Shengli Chen, Guijun Jia, Yuhong Guo, Gangli Zhang, Chunhong Wang, \\ Rui Cheng, Tao Hu, Xuan Zhang, Hongming Ji \\ Department of Neurosurgery, Shanxi Provincial People's Hospital, Taiyuan 030012, China \\ Contributions: (I) Conception and design: X Yan, G Jia, H Ji; (II) Administrative support: H Ji; (III) Provision of study materials or patients: Y Hao, S \\ Chen; (IV) Collection and assembly of data: Y Guo, G Zhang, C Wang; (V) Data analysis and interpretation: X Yan, Y Hao, R Cheng, Tao Hu; (VI) \\ Manuscript writing: All authors; (VII) Final approval of manuscript: All authors. \\ Correspondence to: Guijun Jia; Hongming Ji. Department of Neurosurgery, Shanxi Provincial People's Hospital, No. 29 Shuangtasi Street, Taiyuan \\ 030012, China. Email: JuneAlvarezcan@yahoo.com; gxyg10@163.com.
}

\begin{abstract}
Background: Flavonoids, despite having low nutritional value, have numerous biological activities and extremely beneficial health effects. This study investigated the anticancer activity of rutin in human glioma CHME cells.

Methods: Cytotoxicity was determined through the 3-(4,5-dimethylthiazol-2-yl)-2,5-diphenyltetrazolium bromide (MTT) assay. Protein expression was determined through Western blotting. Apoptosis was detected using annexin V/propidium iodide (PI) and fluorescence microscopy.

Results: Rutin induced maximum cytotoxicity in CHME cells, as revealed through MTT assays. Cell death induced by rutin was due to apoptosis via P53 up-regulation. Rutin induced nuclear condensation, fragmentation, and membrane blebbing, as determined through 4',6-diamidino-2-phenylindole (DAPI) staining. Furthermore, rutin increased reactive oxygen species (ROS) levels and caused a loss of mitochondrial membrane potential, activating the intrinsic apoptotic pathway in CHME cells. The induction of apoptosis by rutin was further confirmed by the release of cytochrome c, up-regulation of BAX, and down-regulation of BCL, activated caspase 9, and caspase 3. The knockdown of P53 reversed rutin-induced apoptosis in a concentration-dependent manner.
\end{abstract}

Conclusions: Rutin plays an important role in the induction of apoptosis in CHME cells. Based on these data, rutin should be further investigated as an anticancer agent in human glioma CHME cells.

Keywords: Apoptosis; CHME cells; mitochondrial membrane potential; reactive oxygen species (ROS); rutin

Submitted Apr 08, 2019. Accepted for publication Aug 13, 2019.

doi: $10.21037 /$ tcr.2019.09.07

View this article at: http://dx.doi.org/10.21037/tcr.2019.09.07

\section{Introduction}

Cancer is the process of unregulated, unlimited cell division and is the second leading cause of death in humans (1). Glioma is considered to be the most aggressive cancer, comprising $50 \%$ of malignant cancers of the central nervous system. Despite various treatments available for glioblastoma patients, the average life expectancy does not exceed one year (2-4). The deadliness of glioblastoma is caused by its ability to deceive the immune system in addition to its high proliferation rate. Effective chemical agents are urgently needed to meet the current demand of patients (5). Despite the discovery of numerous anti-glioma drugs, no effective drug is currently available, as these drugs are transformed into an inactive state in the body or are unable to cross the blood-brain barrier (6). A literature survey showed that numerous edible substances containing various biochemically active components can prevent glioma $(7,8)$. Natural products play a vital role in drug discovery programs. Various anticancer drugs, including doxorubicin, vinblastine, paclitaxel, and camptothecin, 
have been discovered from natural products. Therefore, we selected rutin as a natural flavonoid that could potentially possess anti-tumor and anti-allergy activities, as well as preventing coronary heart, inflammatory bowel, and neurodegenerative diseases (9-11). Rutin acts as a strong antioxidant by chelating iron ions, generating reactive oxygen species (ROS) and thus protecting DNA from oxidative damage (12). Rutin is available in natural products such as vegetables and fruits, but humans are incapable of synthesizing it (13). The induction of apoptosis by rutin has not been reported. Therefore, in this study, we explored the potential of rutin to induce apoptosis in human glioma CHME cells. Apoptosis is a programmed cell death process that eliminates unneeded, damaged, or cancerous cells. It is a genetically programmed and energy-consuming process involving the activation of cysteine proteases called caspases (14). Apoptosis is characterized by cell shrinkage, nuclear segmentation, DNA cleavage, and condensation. Various physiological and pathological signaling insults such as heat, radiation, and hypoxia can trigger apoptosis, and the response to these apoptotic induction signals varies from cell to cell (15). Mitochondria and their membrane potential play an important role in apoptosis induction in all cells. Apoptosis is considered the preferred cell death pathway, rather than necrosis (16), as necrosis involves the inflammatory process, which in turn promotes the growth and metastasis of cancer cells (17). In this study, we explored the effect of rutin on apoptosis in human glioma CHME cells.

\section{Methods}

\section{Chemicals and reagents}

Dulbecco's minimal essential medium (DMEM), streptomycin, penicillin $G$, phosphate-buffered saline, 3-(4,5-dimethylthiazol-2-yl)-2,5-diphenyltetrazolium bromide (MTT), penicillin G, rhodamine 123, 5-diphenyltetrazolium bromide, 5-dimethylthiazole-2yl, dimethyl sulfoxide (DMSO), radioimmunoprecipitation assay (RIPA) buffer, bovine serum albumin (BSA), streptomycin sulfate, 2,7-dichlorodihydrofluorescein diacetate (DCFH-DA), rutin, and 4',6-diamidino-2phenylindole (DAPI) were purchased from Sigma (St. Louis, MO, USA). Fetal bovine serum (FBS) was obtained from Gibco (Grand Island, NY, USA). Polyvinylidene difluoride (PVDF) membranes and Immobilon Chemiluminescent horseradish peroxidase (HRP) substrate were obtained from
Millipore (Burlington, MA, USA). Antibodies, including those against P53, cytochrome c, BAX, BCL2, caspase 3, caspase 9, PARP1, and $\beta$-actin, were purchased from Cell Signaling Technology (Danvers, MA, USA). Annexin V with propidium iodide (PI) was purchased from Santa Cruz Biotechnology (Dallas, TX, USA).

\section{Cell culture and growth conditions}

A panel of six cell lines, including human neuroblastoma SH-SY5Y, mouse neuroblastoma N2a, human glioma CHME cells, human glioblastoma U-118, human glioblastoma LN-229, and human neuroblastoma SK$\mathrm{N}-\mathrm{SH}$, were purchased from the American Type Culture Collection and authenticated through short tandem repeat profiling. These cells were grown in DMEM containing streptomycin and penicillin $\mathrm{G}$ in an incubator at $37^{\circ} \mathrm{C}, 98 \%$ humidity, and $5 \% \mathrm{CO}_{2}$. Cells were subsequently treated with rutin at various concentrations for $24 \mathrm{~h}$.

\section{Cell proliferation assay}

The cell lines listed above were seeded into a 96-well plate treated with rutin at concentrations of $0,5,10$, and $20 \mu \mathrm{M}$ for $24 \mathrm{~h}$. At $4 \mathrm{~h}$ before termination of the experiment, MTT was added to each well. The supernatants were discarded and formazan crystals were dissolved in DMSO. The absorbance was measured using a plate reader at $570 \mathrm{~nm}$.

\section{Colony formation assay}

Human glioblastoma CHME cells were treated with various concentrations of rutin for $24 \mathrm{~h}$. Treated cells were trypsinized and seeded at a density of 1,000 cells per well in 6-well plates. These cells were allowed to form colonies for 21 days. Then, the cells were washed with phosphate buffered saline (PBS) and fixed with $4 \%$ paraformaldehyde for $15 \mathrm{~min}$ followed by staining with $0.1 \%$ crystal violet. Cells were washed three times with PBS and images were taken using an Olympus camera. Colonies were counted using ImageJ software.

\section{ROS}

ROS were measured with a flow cytometer using DCFHDA. Briefly, CHME cells were seeded into 6-well plates at a density of $0.5 \times 10^{6}$ and treated with rutin at various concentrations for $24 \mathrm{~h}$. DCFH-DA $(10 \mu \mathrm{M})$ was added 
to each well $30 \mathrm{~min}$ before termination of the experiment. Cells were washed with PBS followed by trypsinization and then analyzed with a flow cytometer.

\section{Mitochondrial membrane potential}

Mitochondrial membrane potential was analyzed by flow cytometry using rhodamine 123 dye. Cells were seeded in 6-well plates and treated with $0,5,10$, or $20 \mu \mathrm{M}$ rutin for $24 \mathrm{~h}$. Before termination of the experiment, rhodamine 123 was added to each well at a concentration of $200 \mathrm{nM}$. Cells were washed with PBS and analyzed for mitochondrial membrane potential loss through flow cytometry.

\section{Nuclear morphology}

An analysis of nuclear morphology was carried out through fluorescence microscopy with DAPI staining. Rutin at concentrations of $0,5,10$, and $20 \mu \mathrm{M}$ was applied to CHME cells for $24 \mathrm{~h}$. The cells were washed three times with PBS and fixed with methanol for 30 min followed by DAPI staining $(1 \mu \mathrm{g} / \mathrm{mL})$ for $15 \mathrm{~min}$. The cells were then washed and observed via fluorescence microscopy.

\section{Determination of apoptosis using annexin V/PI}

To analyze apoptosis, annexin V/PI staining was conducted and analyzed through flow cytometry. CHME cells were seeded in 6-well plates and treated with the desired concentrations of rutin for $24 \mathrm{~h}$. Next, the cells were resuspended in binding buffer followed by the addition of annexin $\mathrm{V}(5 \mu \mathrm{L})$ and $\mathrm{PI}(3 \mu \mathrm{L})$ into each well for $15 \mathrm{~min}$. Cells were detected using a flow cytometer, with a total of 30,000 events captured.

\section{Western blot analysis}

CHME cells were treated with rutin at various concentrations for the desired time period. They were then collected and lysed in RIPA buffer containing a protease inhibitor cocktail $\left(\mathrm{Na}_{2} \mathrm{VO}_{3}, \mathrm{NaF}\right.$, and PMSF). The samples were centrifuged and their supernatants were collected followed by protein estimation using the Bradford method. Proteins were resolved through polyacrylamide gel electrophoresis and then transferred to PVDF membranes. The membranes were blocked with $5 \%$ skim milk and incubated with primary antibodies overnight at $4{ }^{\circ} \mathrm{C}$. The protein membranes were washed three times using Tris- buffered saline with Tween-20 (TBST) and incubated with HRP-conjugated secondary antibodies for $1 \mathrm{~h}$ at room temperature. The membranes were further washed with TBST and ECL substrate was used to detect signals for capture on X-ray film. Densitometry of the Western blots was carried out using ImageJ software.

\section{Transient transfection of siP53 into CHME cells}

CHME cells were grown in 6-well plates and transfected with siP53 in an OPT: MEM-containing transfection mixture (100 nM siP53 and $5 \mu \mathrm{L}$ of Lipofectamine 3,000 per well) for $12 \mathrm{~h}$. siP53-transfected cells were treated with rutin at various concentrations for $24 \mathrm{~h}$. Cells were collected and lysates were prepared as discussed for Western blotting.

\section{Statistical analysis}

Data are presented here as the mean of three independent experiments, and error bars represent the standard deviation. Comparisons were made between untreated samples and treated samples. Statistical analyses were conducted through analysis of variances (ANOVAs) and $t$-tests with the Bonferroni correction using GraphPad Prism 5.00.288; statistical significance was considered at thresholds of ${ }^{*} \mathrm{P}<0.05,{ }^{* *} \mathrm{P}<0.01$, and ${ }^{* * *} \mathrm{P}<0.005$.

\section{Results}

Inbibition of cell proliferation by rutin in buman glioma CHME cells

To study the effect of rutin on cell proliferation, a panel of cell lines (MDAMB231, CHME, U118, LN18 N2A, and SHSY5Y cells) was treated with rutin at concentrations of $0,5,10$, and $20 \mu \mathrm{M}$ and cell proliferation was calculated (Figure 1A). Among these cell lines, human glioma CHME cells were most sensitive to rutin over $24 \mathrm{~h}$, with a half maximal inhibitory concentration $\left(\mathrm{IC}_{50}\right)$ of $20 \mu \mathrm{M}$ (Figure $1 B$ ). The potential cytotoxicity of rutin in CHME cells prompted further experimental investigation of rutin.

\section{Rutin reduces the clonogenicity of human glioma CHME cells}

To further analyze the effect of rutin on cell growth, a colony formation assay was conducted. CHME cells were treated with rutin at various concentrations. The 


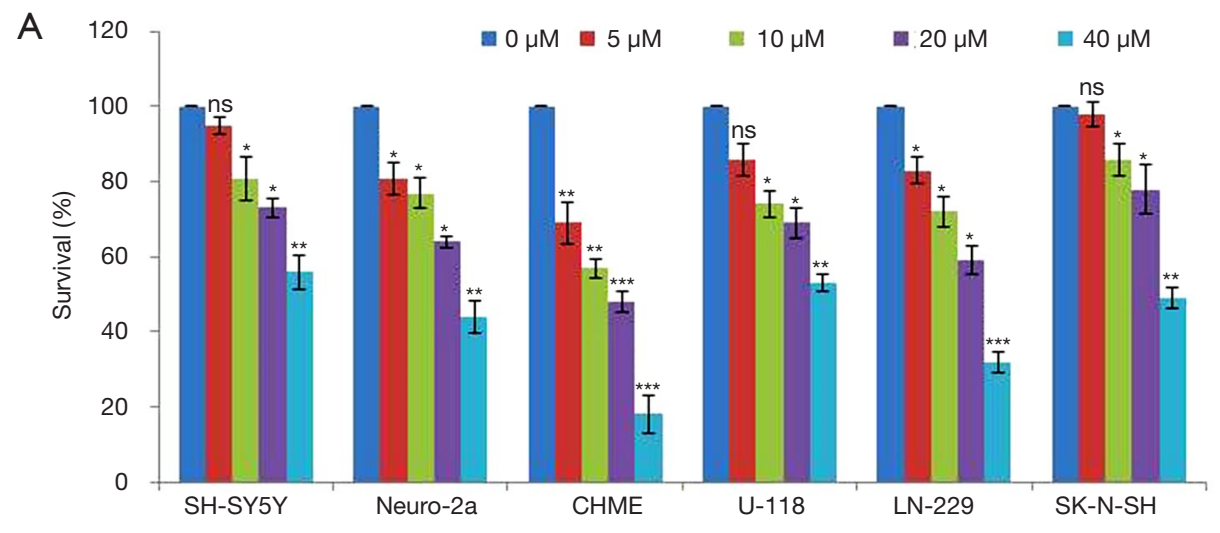

\begin{tabular}{|lll|}
\hline B Serial no. & Cell lines & $\mathrm{IC}_{50}(\mu \mathrm{M})$ \\
\hline 1. & SH-SY5Y & $>40$ \\
\hline 2. & Neuro-2a & $24 \pm 4.3$ \\
\hline 3. & CHME & $15 \pm 2.6$ \\
\hline 4. & $\mathrm{U}-118$ & $>40$ \\
\hline 5. & LN-229 & $22 \pm 4.8$ \\
\hline 6. & SK-N-SH & $36 \pm 3.7$ \\
\hline
\end{tabular}

Figure 1 Rutin-induced cytotoxicity in different cell lines. (A) The viability of different cell lines was inhibited by rutin in a concentrationdependent manner over $24 \mathrm{~h}$; (B) the $\mathrm{IC}_{50}$ values of the cancer cell lines SH-SY5Y, Neuro-2a, CHME, U-118, LN-229, and SK-N-SH were calculated as $>40,24 \pm 4.3,15 \pm 2.6,>40,22 \pm 4.8$, and $36 \pm 3.7 \mu \mathrm{M}$, respectively. The results presented here are the means of three individual experiments. A statistical analysis was carried out using ANOVAs or $t$-tests followed by the Bonferroni correction. Statistical significance was considered at levels of ${ }^{*} \mathrm{P}<0.05,{ }^{* *} \mathrm{P}<0.01$, and ${ }^{* *} \mathrm{P}<0.001$ compared with the untreated control. $\mathrm{IC}_{50}$, half maximal inhibitory concentration; ANOVAs, analysis of variances.

clonogenicity of CHME cells decreased with rutin in a concentration-dependent manner for $24 \mathrm{~h}$ (Figure 2). These results clearly demonstrate that rutin inhibited colony formation in a dose-dependent manner.

\section{Generation of ROS induced by rutin}

The generation of excessive ROS pushes cells toward apoptosis. We found that rutin increased ROS levels in a dose-dependent manner over $24 \mathrm{~h}$. The generation of ROS by rutin was $5 \%, 19 \%, 31 \%$, and $56 \%$ at concentrations of $0,5,10$, and $20 \mu \mathrm{M}$, respectively, in human glioma CHME cells (Figure 3).

\section{Rutin induces apoptosis in CHME cells}

To investigate the mechanism of cell death induced by rutin, apoptosis was measured after treatment with rutin in human glioma CHME cells. Nuclear morphological changes of
CHME treated with rutin at various concentrations were stained with DAPI and visualized using a fluorescence microscope. Interestingly, nuclear condensation, nuclear fragmentation, and membrane blebbing were observed in a concentration-dependent manner compared with untreated cells, which had bright nuclei (Figure 4). These morphological results suggest that rutin induced apoptosis in human glioma CHME cells.

\section{Annexin V/PI detection of apoptosis in CHME cells}

Cell death induced by rutin was confirmed using annexin V/PI, which showed that rutin triggers cell death in a dose-dependent manner compared with the untreated control. The apoptotic populations at concentrations of $0,1,5$, and $10 \mu \mathrm{M}$ were calculated as $0 \%, 5 \%, 20 \%$, and $30 \%$ of cells, respectively (Figure 5). Thus, our results clearly demonstrate that rutin induced apoptosis in human glioma CHME cells. 

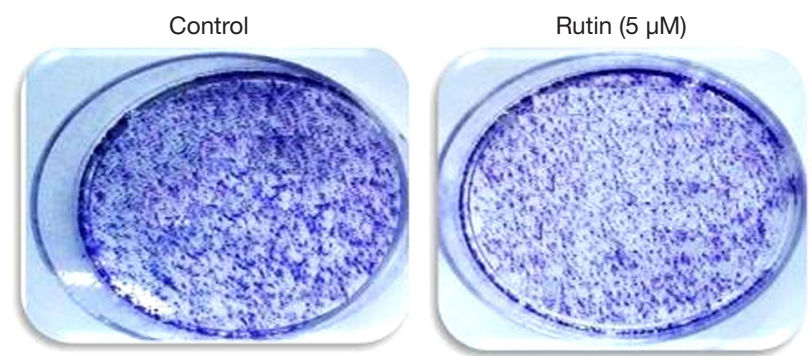

Rutin $(10 \mu \mathrm{M})$

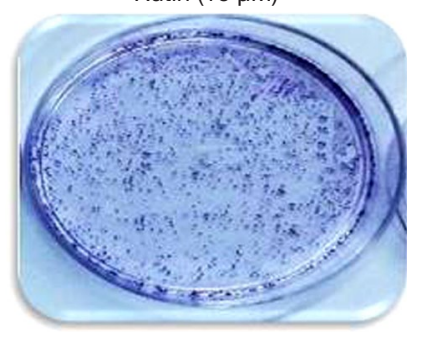

Rutin $(20 \mu \mathrm{M})$
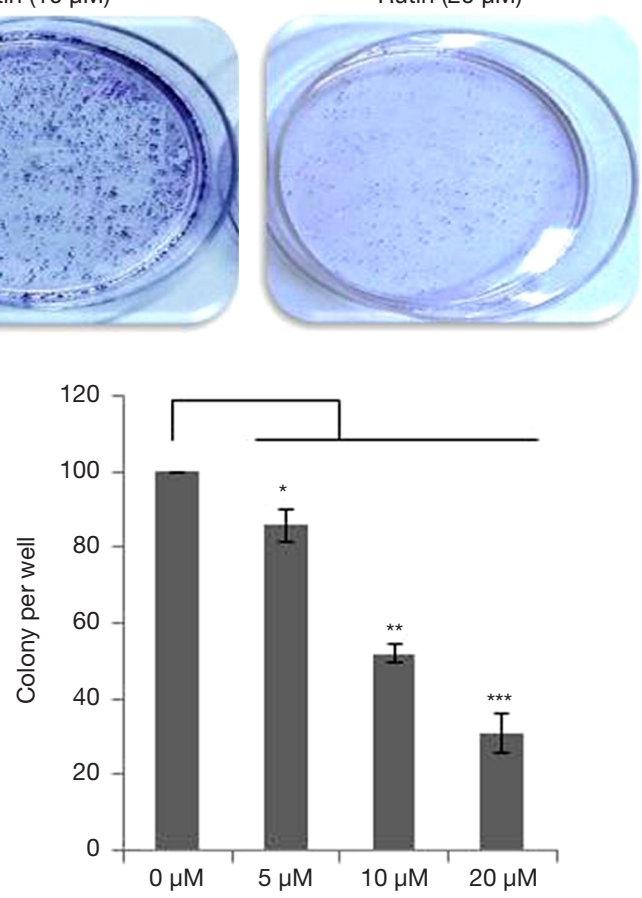

Figure 2 Rutin inhibits colony formation in human glioma CHME cells. Colony formation was inhibited by rutin in a dosedependent manner. The data represent the means \pm standard errors of six experiments performed in triplicate. Untreated samples were compared with rutin-treated samples. The data presented here are the means of three independent experiments; statistical significance was assigned at ${ }^{*} \mathrm{P}<0.05$, ${ }^{* *} \mathrm{P}<0.01$, and ${ }^{* *} \mathrm{P}<0.001$ compared with the untreated control.

\section{Rutin induces apoptosis through the intrinsic pathway in CHME cells}

The intrinsic pathway of apoptosis was analyzed through flow cytometry, which showed that the mitochondrial membrane potential decreased with rutin in a dosedependent manner (Figure 6A). Rutin increased P53 expression in a dose-dependent manner, disrupting the BAX/BCL2 ratio and causing the release of cytochrome $\mathrm{c}$ from the damaged mitochondrial membrane, thereby

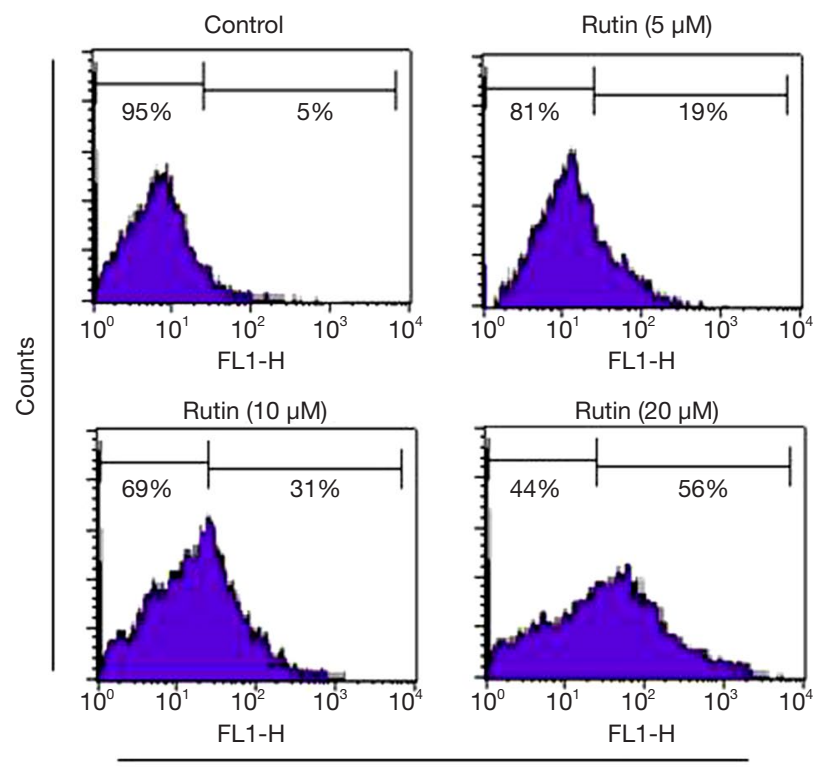

DCFH2DA fluorescence (FL1-H)

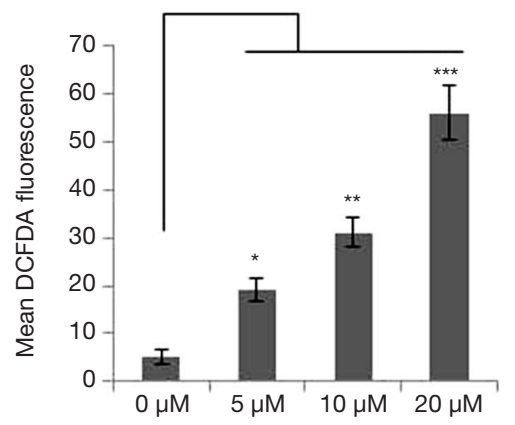

Figure 3 Rutin-induced ROS generation in CHME cells. Flow cytometric analysis of ROS in CHME cells using DCFH-DA. Rutin generated ROS in a concentration-dependent manner in CHME cells. Untreated samples were compared with rutintreated samples. The data presented here are the means of three independent experiments; statistical significance was assigned at ${ }^{*} \mathrm{P}<0.05,{ }^{* *} \mathrm{P}<0.01$, and ${ }^{* *} \mathrm{P}<0.001$ compared with the untreated control. ROS, reactive oxygen species; DCFH-DA, 2,7-dichlorodihydrofluorescein diacetate.

activating caspase 9 and causing the activation of the executional caspase 3. Activated caspase 3 cleaves PARP1, which induces apoptosis by preventing survival signals from reaching the DNA repair pathway and by obstructing energy exertion that would induce necrosis (Figure 6B).

\section{The knockdown of P53 abolishes apoptosis induced by rutin}

To validate the mechanism of apoptosis induced by rutin, 

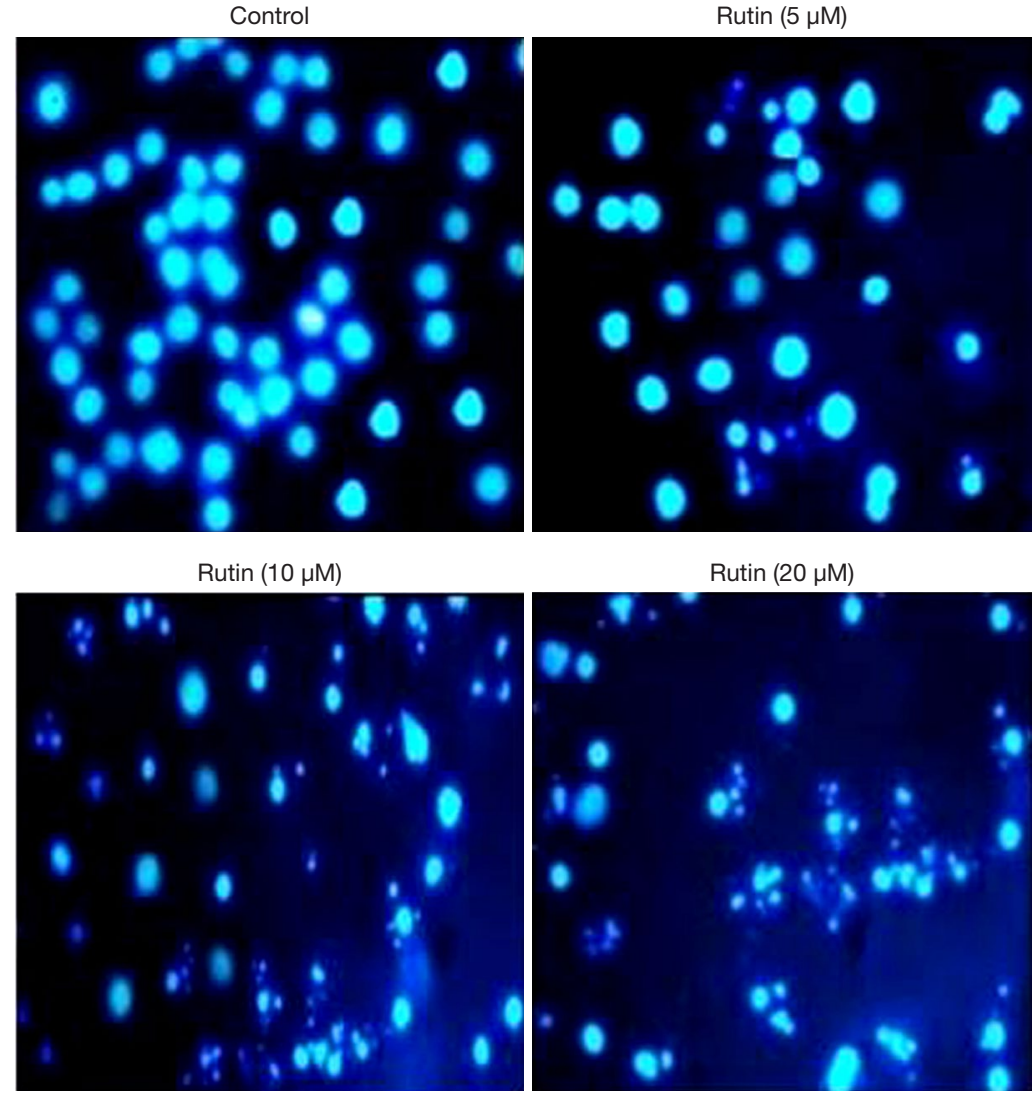

Figure 4 Rutin-induced nuclear morphological changes in CHME cells. Fluorescence microscopic analysis of apoptosis induction in CHME cells using DAPI. Rutin-induced nuclear condensation, nuclear fragmentation, and membrane blebbing were observed in a concentrationdependent manner. The magnification power of fluorescence microscope is 20x. DAPI, 4',6-diamidino-2-phenylindole.
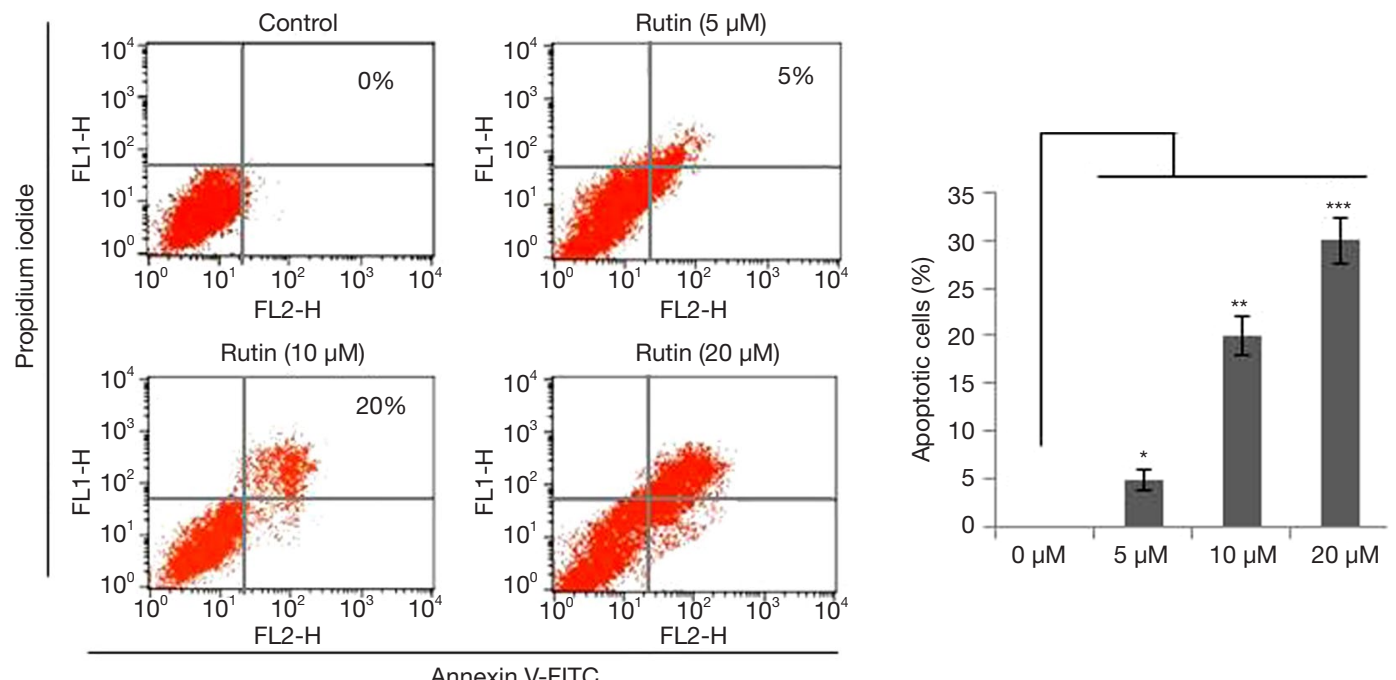

Figure 5 Apoptosis induction by rutin in human glioma CHME cells. Flow cytometric analysis of apoptosis induced by rutin in CHME cells using annexin V/PI. The data presented here are the means of three independent experiments; statistical significance was assigned at ${ }^{*} \mathrm{P}<0.05,{ }^{* *} \mathrm{P}<0.01$, and ${ }^{* * *} \mathrm{P}<0.001$ compared with the untreated control. $\mathrm{PI}$, propidium iodide. 
A


0
$\stackrel{0}{5}$
0
0
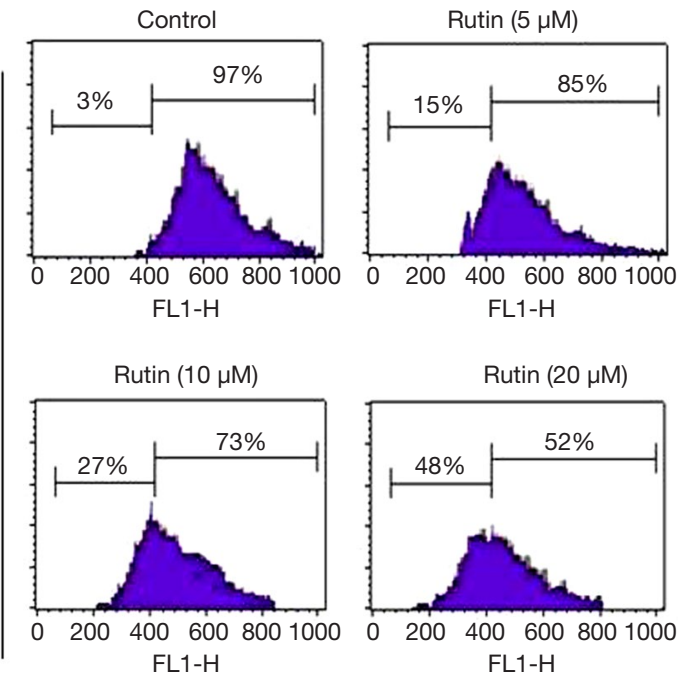

B

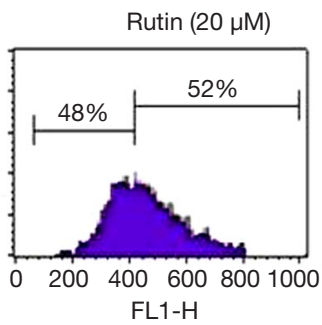

Rhodamine-123 fluorescence

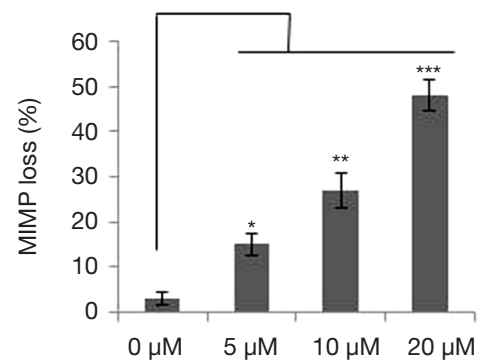

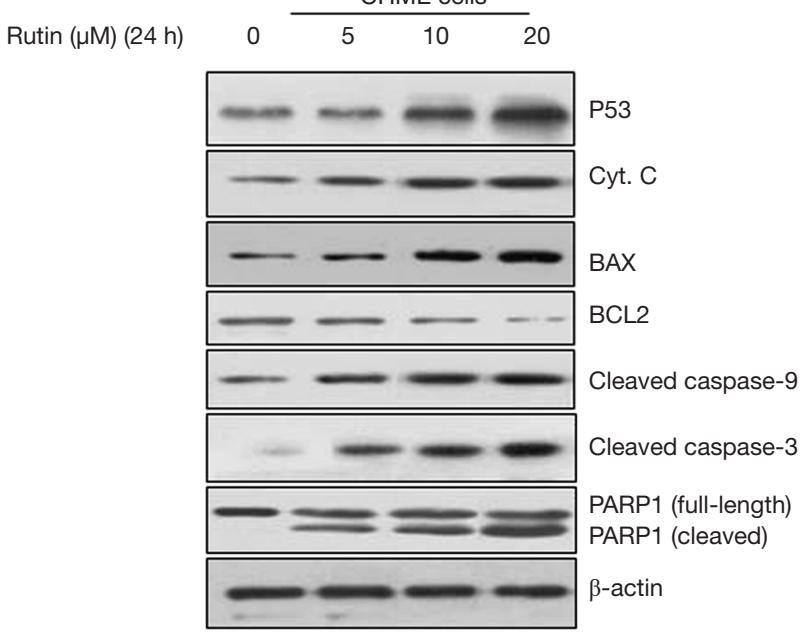

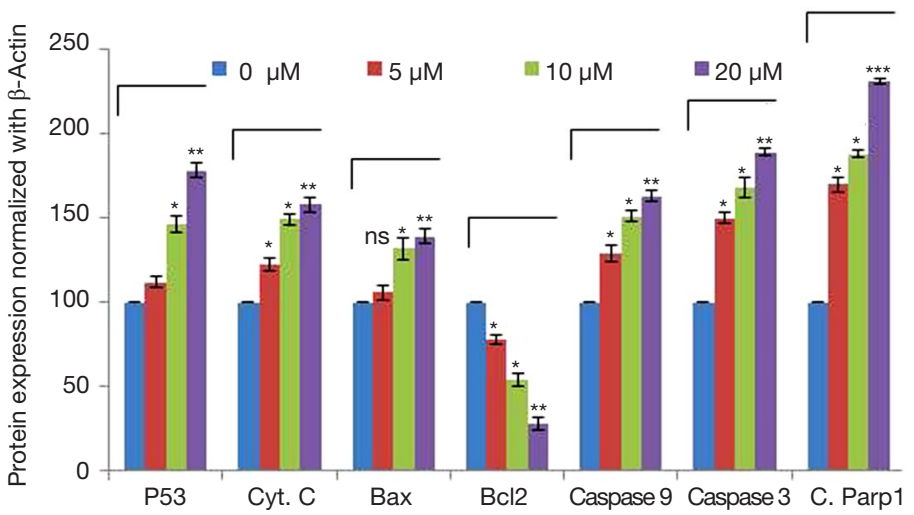

Figure 6 Rutin-induced mitochondrial membrane potential loss and apoptosis in CHME cells. (A) Flow cytometric analysis showed that rutin induced mitochondrial membrane potential loss in a dose-dependent manner; (B) western blotting showed that rutin up-regulated P53, BAX, cytochrome c, and the activation of caspase 9 and caspase 3 and also down-regulated BCL2 and PARP1 cleavage in CHME cells, all in a dose-dependent manner. The data presented here are the means of three independent experiments; statistical significance was assigned at ${ }^{*} \mathrm{P}<0.05,{ }^{* *} \mathrm{P}<0.01$, and ${ }^{* * *} \mathrm{P}<0.001$ compared with the untreated control.

CHME cells were transfected with siP53 followed by rutin treatment. The siP53 CHME cells exhibited reduced rutin-induced apoptosis in a concentration-dependent manner. The expression of P53, cytochrome c, BAX, BCL2, caspase 3, and caspase 9 remained unchanged (Figure $7 A$ ). The viability of the siP53 CHME cells was unaffected by treatment with rutin for $24 \mathrm{~h}$, as determined through MTT assays (Figure $7 B$ ). The efficacy of siP53 was compared with the control through Western blot analysis (Figure 7C).

\section{Discussion}

Radiotherapy and surgery have been effective for curing earlystage glioblastoma, but no therapy is available for advanced glioma (18). Chemotherapy offers a mechanism to cure various cancers, with the possibility of curbing the chronic stage of glioblastoma and increasing the life expectancy of patients (19). Flavonoids have shown promise as a therapeutic target against glioma (20), and a literature survey suggested that a flavonoid-enriched diet could reduce glioma frequency, metastasis, and progression (21). Hence, we investigated rutin as a potential anticancer agent against glioblastoma. Previously, it was revealed that rutin caused anticancer activity against neuroblastoma $(7,22)$ through the induction of various tumor suppressor pathways. We established the molecular mechanism of rutin activity in a concentration-dependent manner in CHME cells. Rutin induces apoptosis through the up-regulation of P53, which is known as the guardian of 
A

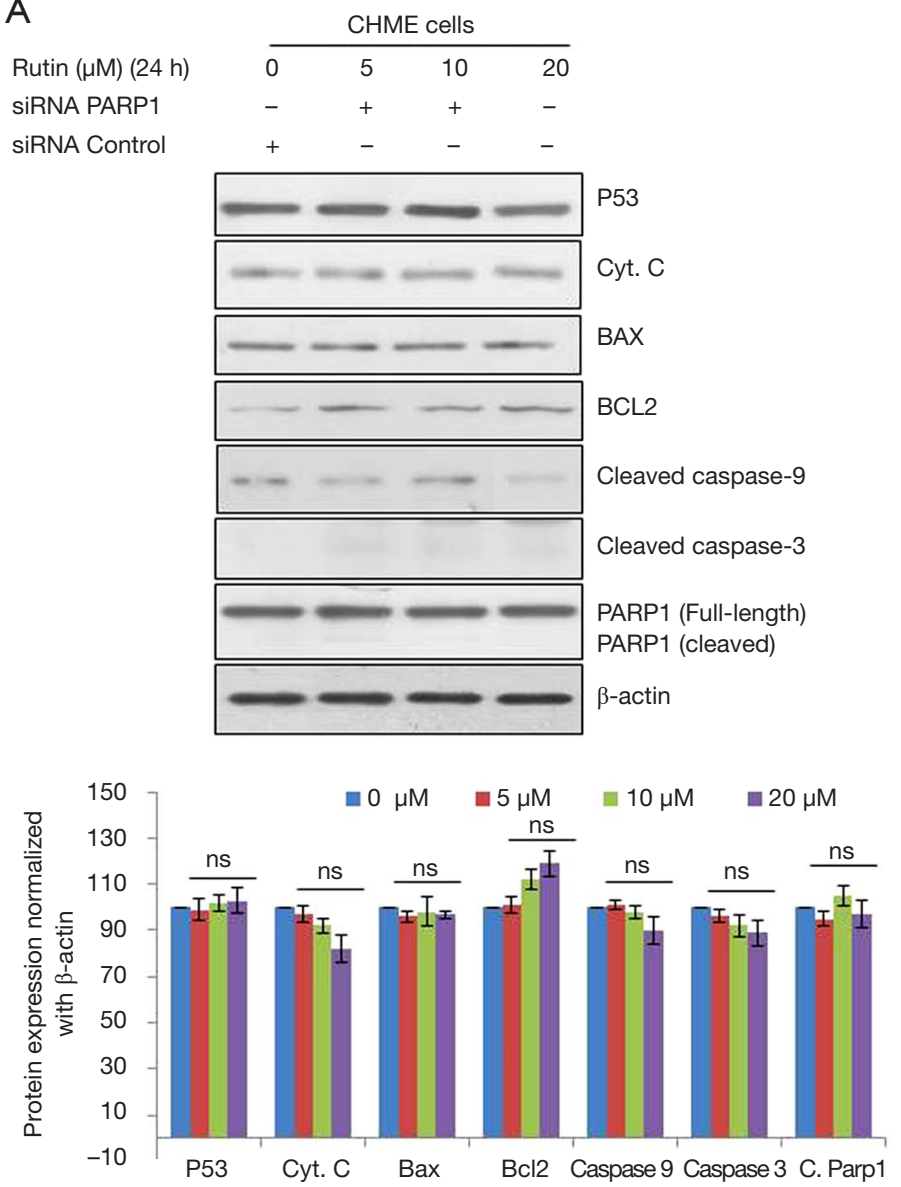

B

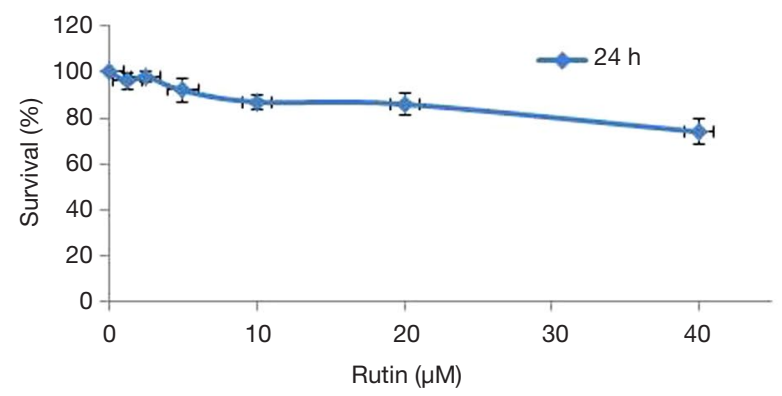

$\begin{array}{llll}\text { siP53 } & - & + \\ \text { si Mock } & + & -\end{array}$
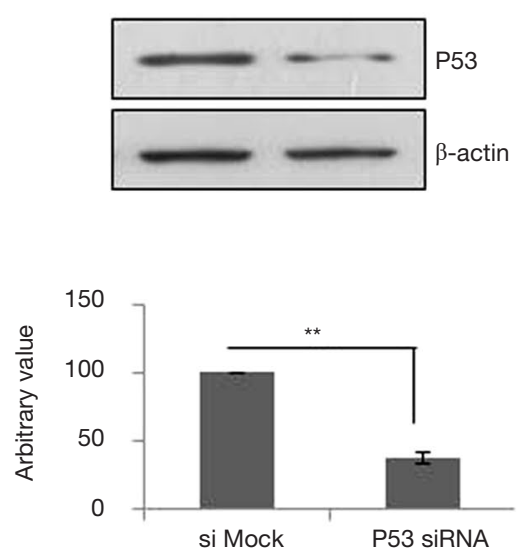

Figure 7 The knockdown of P53 abolishes the effect of rutin in human glioma CHME cells. (A) Western blot analysis of P53, cytochrome c, BAX, BCL2, caspase 9, caspase 3, and PARP1 in knockdown P53 CHME cells after treatment with various concentrations of rutin; (B) cell viability was calculated in knockdown P53 CHME cells treated with rutin; (C) western blotting showed the efficacy of siP53 in CHME cells. The data presented here are the means of three independent experiments; statistical significance was assigned at ${ }^{*} \mathrm{P}<0.05,{ }^{* *} \mathrm{P}<0.01$, and ${ }^{* * *} \mathrm{P}<0.001$ compared with the untreated control.

the genome (23). P53 activation promotes ROS generation, leading cells toward apoptosis. Interestingly, mitochondrial membrane potential loss due to rutin triggers the intrinsic apoptotic pathway by altering the BAX/BCL2 ratio and releasing cytochrome $\mathrm{c}$ into the cytosol, which in turn causes the activation of caspase 9 and caspase 3. This cascade of events leads human glioma CHME cells toward apoptosis.

In conclusion, the present findings show that rutin inhibits cell proliferation, activates apoptosis by inducing the loss of mitochondrial membrane potential, up-regulating ROS generation, and activating the mitochondrial intrinsic apoptotic pathway in human glioma CHME cells. In vivo validation and further investigation to explore the molecular mechanism employed by rutin are needed.

\section{Acknowledgments}

Funding: This work was supported by the Scientific Research Topics of Shanxi Province Health Commission, China (Project No. 2017021).

\section{Footnote}

Conflicts of Interest: All authors have completed the ICMJE uniform disclosure form (available at http://dx.doi. org/10.21037/tcr.2019.09.07). The authors have no conflicts of interest to declare.

Ethical Statement: The authors are accountable for all aspects of the work in ensuring that questions related 
to the accuracy or integrity of any part of the work are appropriately investigated and resolved. This study was conducted in accordance with the Declaration of Helsinki (as revised in 2013). The study was approved by the ethics committee of Shanxi Provincial People's Hospital (No. TXY20160402). Individual informed consent was waived.

Open Access Statement: This is an Open Access article distributed in accordance with the Creative Commons Attribution-NonCommercial-NoDerivs 4.0 International License (CC BY-NC-ND 4.0), which permits the noncommercial replication and distribution of the article with the strict proviso that no changes or edits are made and the original work is properly cited (including links to both the formal publication through the relevant DOI and the license). See: https://creativecommons.org/licenses/by-nc-nd/4.0/.

\section{References}

1. DeAngelis LM. Brain tumors. N Engl J Med 2001;344:114-23.

2. Lamborn KR, Yung WK, Chang SM, et al. Progression-free survival: an important end point in evaluating therapy for recurrent high-grade gliomas. Neuro Oncol 2008;10:162-70.

3. Stupp R, Mason WP, van den Bent MJ, et al. Radiotherapy plus concomitant and adjuvant temozolomide for glioblastoma. N Engl J Med 2005;352:987-96.

4. Ballman KV, Buckner JC, Brown PD, et al. The relationship between six-month progression-free survival and 12-month overall survival end points for phase II trials in patients with glioblastoma multiforme. Neuro Oncol 2007;9:29-38.

5. Curtin JF, King GD, Candolfi M, et al. Combining cytotoxic and immune-mediated gene therapy to treat brain tumors. Curr Top Med Chem 2005;5:1151-70.

6. Lee YS, Jin DQ, Kwon EJ, et al. Asiatic acid, a triterpene, induces apoptosis through intracellular $\mathrm{Ca} 2$ + release and enhanced expression of p53 in HepG2 human hepatoma cells. Cancer Lett 2002;186:83-91.

7. Anand P, Kunnumakkara AB, Sundaram C, et al. Cancer is a preventable disease that requires major lifestyle changes. Pharm Res 2008;25:2097-116.

8. Khan N, Afaq F, Mukhtar H. Cancer chemoprevention through dietary antioxidants: progress and promise. Antioxid Redox Signal 2008;10:475-510.

9. Kahraman A, Erkasap N, Serteser M, et al. Protective effect of quercetin on renal ischemia/reperfusion injury in rats. J Nephrol 2003;16:219-24.

10. de Whalley CV, Rankin SM, Hoult JR, et al. Flavonoids inhibit the oxidative modification of low density lipoproteins by macrophages. Biochem Pharmacol 1990;39:1743-50.

11. McAnulty SR, McAnulty LS, Nieman DC, et al. Chronic quercetin ingestion and exercise-induced oxidative damage and inflammation. Appl Physiol Nutr Metab 2008;33:254-62.

12. Valério DA, Georgetti SR, Magro DA, et al. Quercetin reduces inflammatory pain: inhibition of oxidative stress and cytokine production. J Nat Prod 2009;72:1975-9.

13. Scholz S, Williamson $G$. Interactions affecting the bioavailability of dietary polyphenols in vivo. Int J Vitam Nutr Res 2007;77:224-35.

14. Geske FJ, Gerschenson LE. The biology of apoptosis. Hum Pathol 2001;32:1029-38.

15. Li WX, Franklin WA. Radiation- and heat-induced apoptosis in PC-3 prostate cancer cells. Radiat Res 1998;150:190-4.

16. Snider BJ, Gottron FJ, Choi DW. Apoptosis and necrosis in cerebrovascular disease. Ann N Y Acad Sci 1999;893:243-53.

17. Abd-Elrahman I, Hershko K, Neuman T, et al. The inhibitor of apoptosis protein Livin (ML-IAP) plays a dual role in tumorigenicity. Cancer Res 2009;69:5475-80.

18. Lokker NA, Sullivan CM, Hollenbach SJ, et al. Plateletderived growth factor (PDGF) autocrine signaling regulates survival and mitogenic pathways in glioblastoma cells: evidence that the novel PDGF-C and PDGF-D ligands may play a role in the development of brain tumors. Cancer Res 2002;62:3729-35.

19. Chen J, Li Y, Yu TS, et al. A restricted cell population propagates glioblastoma growth after chemotherapy. Nature 2012;488:522-6.

20. Das A, Banik NL, Ray SK. Flavonoids activated caspases for apoptosis in human glioblastoma T98G and U87MG cells but not in human normal astrocytes. Cancer 2010;116:164-76.

21. Shen SC, Lin CW, Lee HM, et al. Lipopolysaccharide plus 12-o-tetradecanoylphorbol 13-acetate induction of migration and invasion of glioma cells in vitro and in vivo: Differential inhibitory effects of flavonoids. Neuroscience 2006;140:477-89.

22. Tian X, Li F, Zhu L, et al. Study on the electrochemical behavior of anticancer herbal drug rutin and its interaction with DNA. J Electroanal Chem 2008, 621:1-6.

23. Efeyan A, Serrano M. p53: guardian of the genome and policeman of the oncogenes. Cell Cycle 2007;6:1006-10.

Cite this article as: Yan X, Hao Y, Chen S, Jia G, Guo Y, Zhang G, Wang C, Cheng R, Hu T, Zhang X, Ji H. Rutin induces apoptosis via P53 up-regulation in human glioma CHME cells. Transl Cancer Res 2019;8(5):2005-2013. doi: 10.21037/tcr.2019.09.07 